\title{
Extended Quantum Conditional Entropy and Quantum Uncertainty Inequalities
}

\author{
Rupert L. Frank ${ }^{1}$, Elliott H. Lieb ${ }^{2}$ \\ 1 Department of Mathematics, Princeton University, Washington Road, Princeton, NJ 08544, USA. \\ E-mail: rlfrank@math.princeton.edu \\ 2 Departments of Mathematics and Physics, Princeton University, Washington Road, Princeton, NJ 08544, \\ USA. E-mail: lieb@Math.Princeton.EDU
}

Received: 5 June 2012 / Accepted: 3 January 2013

Published online: 11 August 2013 - (C) The Author(s) 2013

\begin{abstract}
Quantum states can be subjected to classical measurements, whose incompatibility, or uncertainty, can be quantified by a comparison of certain entropies. There is a long history of such entropy inequalities between position and momentum. Recently these inequalities have been generalized to the tensor product of several Hilbert spaces and we show here how their derivations can be shortened to a few lines and how they can be generalized. Our proofs utilize the technique of the original derivation of strong subadditivity of the von Neumann entropy.
\end{abstract}

\section{Introduction}

In this paper we prove certain inequalities about quantum entropies for states defined over the product of several spaces. The inherent difficulties in proving these theorems usually involve some kind of entanglement of the several spaces. Some of our results are new and some show how previously derived results can be more easily obtained by going back to the original proof technique used to establish the strong subadditivity of von Neumann entropy in [1].

We start by recalling the celebrated inequality of Maassen-Uffink [2], based on earlier work by Hirschman [3], Deutsch [4], and Kraus [5], which relates the 'classical' entropies of a quantum density matrix in two different bases and shows that although one of them could be very small, the sum of the two is bounded below by a positive constant. The word 'classical' refers to $H(A)=-\sum_{j} p_{j} \ln p_{j}$ and $H(B)=-\sum_{k} q_{k} \ln q_{k}$, where $p_{j}=\left\langle a_{j}, \rho a_{j}\right\rangle$ and $q_{k}=\left\langle b_{k}, \rho b_{k}\right\rangle$ are the expectations of a density matrix $\rho$ in two orthonormal bases $A=\left(a_{j}\right)$ and $B=\left(b_{k}\right)$. The inequality is

$$
H(A)+H(B) \geq-2 \ln \sup _{j, k}\left|\left\langle a_{j}, b_{k}\right\rangle\right| .
$$

(C) 2013 by the authors. This paper may be reproduced, in its entirety, for non-commercial purposes. 
This can be generalized to continuous bases, like position $\mathbf{x}$ and momentum $\mathbf{p}$, in which case the inequality becomes (with $\mathbf{p}=2 \pi \mathbf{k}$ )

$$
-\int_{\mathbb{R}^{d}} d^{d} \mathbf{x} \rho(\mathbf{x}, \mathbf{x}) \ln \rho(\mathbf{x}, \mathbf{x})-\int_{\mathbb{R}^{d}} d^{d} \mathbf{k} \widehat{\rho}(\mathbf{k}, \mathbf{k}) \ln \widehat{\rho}(\mathbf{k}, \mathbf{k}) \geq 0 .
$$

Here, $\widehat{\rho}$ is the Fourier transform of $\rho$, defined by

$$
\widehat{\rho}(\mathbf{k}, \mathbf{k})=\iint_{\mathbb{R}^{d} \times \mathbb{R}^{d}} d^{d} \mathbf{x} d^{d} \mathbf{x}^{\prime} e^{-2 \pi i\left(\mathbf{k} \cdot \mathbf{x}-\mathbf{k}^{\prime} \cdot \mathbf{x}^{\prime}\right)} \rho\left(\mathbf{x}, \mathbf{x}^{\prime}\right) .
$$

Note that the individual entropies in (2), unlike those in (1), can be arbitrarily negative. A very negative entropy corresponds to a very sharp distribution of the density and hence (2) says that the position space and momentum space densities cannot both be sharp.

These inequalities have subsequently been improved; see the review [6] and the recent papers [7-10]. We emphasize that only purely classical analysis is needed to prove these inequalities, and only one Hilbert space is involved.

Another direction was opened up by the conjectures of Renes and Boileau [11], in which more than one Hilbert space appears. The analogous inequalities become more difficult mathematically because of the well-known entanglement problems in quantum mechanics. Indeed, as noted in [11], the Lieb-Ruskai strong subadditivity (SSA) theorem [1], or one of its equivalents, would ultimately be needed to prove the conjectures. (In some recent papers SSA is called data processing inequality.) Berta et al. [12] then proved a special 2-space version of the conjecture, that can be called the rankone version, and used [1] again (this time the concavity of conditional entropy, which is equivalent to SSA) to deduce a 3-space version of the uncertainty principle. This quantum version of the uncertainty principle has attracted a great deal of attention.

Subsequently, Coles et al. [7] and Tomamichel and Renner [13] were able to eliminate the rank one condition for the 3-space version and partially removed it for the 2-space version. In [14], Coles et al. extended the 3-space version to a larger class of entropies than the von Neumann entropy. Indeed, it is noteworthy that their proof relies only on some basic axioms of entropy, the most important of which is monotonicity of relative entropy, which is equivalent to SSA $[15,16]$. The significant developments in $[7,12]$ came at the cost of rather lengthy calculations and multi-page proofs.

It is evidently desirable to find proofs that are short and unify the previous results for the von Neumann entropy. It is shown here that if one uses the original proof structure of SSA [1] the aforementioned proofs can be significantly shortened and stronger results can be obtained. In particular, we eliminate the restriction in [7] in the 2-space case. Although Coles et al. [7] point out that the obvious extension is necessarily false, we succeed in finding a formulation that makes the extension possible.

These new proofs and results, which can be viewed as quantum extensions of the discrete Maassen-Uffink inequality (1), are described in Sect. 2 and proved in Sect. 3.

In Sect. 4 we revisit the continuous Maassen-Uffink inequality (2) and relate the $\mathbf{x}$-space entropy to the $\mathbf{p}$-space entropy, but this time with an auxiliary quantum system, thereby promoting the Maassen-Uffink inequality to a truly quantum one. This family of results is novel and is easily obtained from the mathematical apparatus of Sects. 2 and 3.

\section{Discrete Measurements on Two and Three Spaces}

The notation in this field is not uniform and we begin by defining our terms. We have three Hilbert spaces (degrees of freedom) $\mathcal{H}_{1}, \mathcal{H}_{2}$ and $\mathcal{H}_{3}$ and their tensor products 
$\mathcal{H}_{123}=\mathcal{H}_{1} \otimes \mathcal{H}_{2} \otimes \mathcal{H}_{3}, \mathcal{H}_{12}=\mathcal{H}_{1} \otimes \mathcal{H}_{2}$ etc. We have density matrices $\rho_{123}, \rho_{12}$, etc. (i.e., non-negative operators of trace one). If $\rho_{123}$ is defined on $\mathcal{H}_{123}$ then there is a natural $\rho_{12}$ on $\mathcal{H}_{12}$ given by $\rho_{12}=\operatorname{Tr}_{3} \rho_{123}$, where $\operatorname{Tr}_{3}$ is the partial trace over $\mathcal{H}_{3}$. In general, entropy is defined by $S(\rho)=-\operatorname{Tr}_{\mathcal{H}} \rho \ln \rho$, where $\mathcal{H}$ is one of the above spaces. If $\rho$ and $\mathcal{H}$ are understood, then $S_{12}=S\left(\rho_{12}\right)$ and $S_{1}=S\left(\rho_{1}\right)$. Conditional entropy is defined by

$$
S(1 \mid 2)=S_{12}-S_{2}=S\left(\rho_{12}\right)-S\left(\rho_{2}\right)
$$

and, as shown in [1], this is a concave function of $\rho_{12}$. This concavity is mathematically equivalent to SSA, and we use it in Lemma 4 below.

A classical measurement on $\mathcal{H}_{1}$ is defined by a sequence of operators $A_{1}, A_{2}$, etc. on $\mathcal{H}_{1}$ such that $\sum_{j} A_{j}^{\dagger} A_{j}=1$. We will need two measurements in our discussion, so we will need a second sequence $B_{1}, B_{2}$, etc. on $\mathcal{H}_{1}$ such that $\sum_{k} B_{k}^{\dagger} B_{k}=1$. At the end of this paper we will let the indices $j$ and $k$ be continuous and the sums become integrals, but for simplicity we stay with sums for now. A measurement $A$ is called 'rank-one' if every $A_{j}$ is a rank-one matrix, namely, $A_{j}=\left|a_{j}\right\rangle\left\langle a_{j}\right|$.

The classical/quantum conditional entropy corresponding to $A$ and $\rho$ is

$$
H\left(1^{A} \mid 2\right)=-\sum_{j} \operatorname{Tr}_{2}\left(\operatorname{Tr}_{1} A_{j} \rho_{12} A_{j}^{\dagger}\right) \ln \left(\operatorname{Tr}_{1} A_{j} \rho_{12} A_{j}^{\dagger}\right)-S\left(\rho_{2}\right) .
$$

Note where the summation sits. (The quantity $H\left(1^{A} \mid 2\right)$ is not the conditional entropy of $\sum_{j} A_{j} \rho A_{j}^{\dagger}$. It is known, however, to be the conditional entropy in an artificially enlarged space; see the proof of Lemma 4.) The quantity $H\left(1^{B} \mid 2\right)$ is defined similarly.

Theorem 1 (2 spaces). For any density matrix $\rho_{12}$,

$$
H\left(1^{A} \mid 2\right)+H\left(1^{B} \mid 2\right) \geq S(1 \mid 2)-2 \ln c_{1},
$$

where $c_{1}=\sup _{j, k} \sqrt{\operatorname{Tr}_{1} B_{k} A_{j}^{\dagger} A_{j} B_{k}^{\dagger}}$.

In the special case that $\rho_{12}=\rho_{1} \otimes \rho_{2}$ is a product state one easily sees that (3) reduces to the 1-space theorems in [7-9], which extend the Maassen-Uffink theorem.

Theorem 1 is new. Coles et al. [7] prove (3) under the additional condition that either $A$ or $B$ is rank-one. They state the result with the constant

$$
c_{\infty}=\sup _{j, k} \sqrt{\left\|B_{k} A_{j}^{\dagger} A_{j} B_{k}^{\dagger}\right\|_{\infty}}
$$

which, however, coincides with $c_{1}$ under the rank one assumption (see next paragraph). In general, $c_{1} \geq c_{\infty}$. As stated in [7], the theorem cannot hold for general $A$ and $B$ in the $c_{\infty}$ version. As we shall show, however, it does hold with the constant $c_{1}$. Thus, we improve on the theorem of Coles et al. [7] by eliminating the rank-one condition.

The norm $\left\|B_{k} A_{j}^{\dagger} A_{j} B_{k}^{\dagger}\right\|_{\infty}$ is the largest eigenvalue of $B_{k} A_{j}^{\dagger} A_{j} B_{k}^{\dagger}$, which is also the largest eigenvalue of $\sqrt{B_{k}^{\dagger} B_{k}} A_{j}^{\dagger} A_{j} \sqrt{B_{k}^{\dagger} B_{k}}$, and thus coincides with $\left\|\sqrt{A_{j}^{\dagger} A_{j}} \sqrt{B_{k}^{\dagger} B_{k}}\right\|_{\infty}^{2}$, which appears in [7]. This shows that our $c_{\infty}^{2}$ is, indeed, the same as that in [7, Eq. (57)].

Our next theorem concerns three spaces. 
Theorem 2 (3 spaces, [7]). For any density matrix $\rho_{123}$,

$$
H\left(1^{A} \mid 2\right)+H\left(1^{B} \mid 3\right) \geq-2 \ln c_{\infty} .
$$

We shall prove the 3 -space theorem by first proving the following theorem which is not conveniently expressed in the $H(\cdot \mid \cdot)$ notation.

Theorem 3 ( $2 \frac{1}{2}$ spaces). For any density matrix $\rho_{12}$,

$$
H\left(1^{A} \mid 2\right)-\sum_{k} \operatorname{Tr}_{12} B_{k} \rho_{12} B_{k}^{\dagger} \ln B_{k} \rho_{12} B_{k}^{\dagger}-S\left(\rho_{12}\right) \geq-2 \ln c_{\infty} .
$$

This looks like another 2-space theorem but, as we explain below, it is really the 3 -space theorem applied to a pure state (i.e., rank-one) $\rho_{123}$. Thus our title ' $2 \frac{1}{2}$ space'. We shall prove Theorem 3 first and then derive Theorem 2 from it by the concavity of conditional entropy [1]. We repeat that the main point of our proofs of Theorems 2 and 3 is their directness.

\section{Proofs of Theorems 1,2 and 3}

The key inequality behind the proofs of all our three theorems is the three operator generalization of the Golden-Thompson inequality in [15], from which SSA follows as a corollary, as in the original proof [1]. It states that for non-negative operators $X, Y, Z$,

$$
\operatorname{Tr} e^{\ln X-\ln Y+\ln Z} \leq \int_{0}^{\infty} d t \operatorname{Tr} X(Y+t)^{-1} Z(Y+t)^{-1}
$$

Note that in the special case $Z=1$ this reduces to the classical Golden-Thompson inequality. This inequality was a byproduct of the proof of concavity of the generalized Wigner-Yanase skew information.

We shall also need the Gibbs variational principle (equivalent to the Peierls-Bogolubov inequality)

$$
\operatorname{Tr} \rho h-S(\rho) \geq-\ln \operatorname{Tr} e^{-h}
$$

for any density matrix $\rho$ and any self-adjoint $h$ (see, e.g., [17]), and the Davis operator Jensen inequality,

$$
\sum_{j} K_{j}^{\dagger}\left(\ln T_{j}\right) K_{j} \leq \ln \left(\sum_{j} K_{j}^{\dagger} T_{j} K_{j}\right)
$$

for any positive operators $T_{j}$ and any $K_{j}$ with $\sum_{j} K_{j}^{\dagger} K_{j}=1$ (see [18] and also [17]).

Proof of Theorem 1 (2-space theorem). We use (6) for both $A$ and $B$ to bound

$$
H\left(1^{A} \mid 2\right)+H\left(1^{B} \mid 2\right)-S(1 \mid 2) \geq \operatorname{Tr}_{12} \rho_{12} h-S\left(\rho_{12}\right),
$$

with the operator

$$
h=\mathbb{1} \otimes \ln \rho_{2}-\ln \sum_{j} A_{j}^{\dagger} A_{j} \otimes\left(\operatorname{Tr}_{1} A_{j} \rho_{12} A_{j}^{\dagger}\right)-\ln \sum_{k} B_{k}^{\dagger} B_{k} \otimes\left(\operatorname{Tr}_{1} B_{k} \rho_{12} B_{k}^{\dagger}\right) .
$$

We do not need to invoke (6) when $A$ and $B$ are rank-one; in that case (7) is an equality and the proof simplifies further. 
Thus, by (7) and (5),

$$
H\left(1^{A} \mid 2\right)+H\left(1^{B} \mid 2\right)-S(1 \mid 2) \geq-\ln \operatorname{Tr}_{12} e^{-h},
$$

and it remains to show that $\operatorname{Tr}_{12} e^{-h} \leq c_{1}^{2}$. Now comes the crucial step! We use (4) to bound

$$
\operatorname{Tr}_{12} e^{-h} \leq \int_{0}^{\infty} d t \sum_{j, k} \operatorname{Tr}_{12} C_{j, k}(t)
$$

with

$$
\begin{aligned}
C_{j, k}(t) & =A_{j}^{\dagger} A_{j}\left(\operatorname{Tr}_{1} A_{j} \rho_{12} A_{j}^{\dagger}\right)\left(\rho_{2}+t\right)^{-1} B_{k}^{\dagger} B_{k}\left(\operatorname{Tr}_{1} B_{k} \rho_{12} B_{k}^{\dagger}\right)\left(\rho_{2}+t\right)^{-1} \\
& =A_{j}^{\dagger} A_{j} B_{k}^{\dagger} B_{k} \otimes D_{j, k}(t) .
\end{aligned}
$$

Here, $D_{j, k}(t)$ is the operator on $\mathcal{H}_{2}$ given by

$$
\left(\operatorname{Tr}_{1} A_{j} \rho_{12} A_{j}^{\dagger}\right)\left(\rho_{2}+t\right)^{-1}\left(\operatorname{Tr}_{1} B_{k} \rho_{12} B_{k}^{\dagger}\right)\left(\rho_{2}+t\right)^{-1} .
$$

Thus,

$$
\operatorname{Tr}_{12} C_{j, k}(t)=\left(\operatorname{Tr}_{1} A_{j}^{\dagger} A_{j} B_{k}^{\dagger} B_{k}\right) \operatorname{Tr}_{2} D_{j, k}(t) \leq c_{1}^{2} \operatorname{Tr}_{2} D_{j, k}(t) .
$$

We next note that $\sum_{j} \operatorname{Tr}_{1} A_{j} \rho_{12} A_{j}^{\dagger}=\sum_{j} \operatorname{Tr}_{1} A_{j}^{\dagger} A_{j} \rho_{12}=\rho_{2}$, and similarly for the $k$ sum, and obtain

$$
\sum_{j, k} \operatorname{Tr}_{2} D_{j, k}(t)=\operatorname{Tr}_{2} \rho_{2}^{2}\left(\rho_{2}+t\right)^{-2} .
$$

Thus, since $\int_{0}^{\infty} d t\left(\rho_{2}+t\right)^{-2}=\rho_{2}^{-1}$,

$$
\int_{0}^{\infty} d t \sum_{j, k} \operatorname{Tr}_{2} D_{j, k}(t)=\operatorname{Tr}_{2} \rho_{2}=1 .
$$

This completes the proof of the 2-space theorem.

We shall now show that the 3 -space theorem is a corollary of the $2 \frac{1}{2}$-space theorem, so that the proof of the $2 \frac{1}{2}$-space theorem will finish everything. We use the following:

Lemma 4. $H\left(1^{A} \mid 2\right)$ is a concave function on the set of non-negative operators $\rho_{12}$ on $\mathcal{H}_{12}$.

Proof. The idea is to view the sum over $j$ as the trace over an auxiliary space $\mathcal{K}$ of a matrix that happens to be diagonal in this space, and to apply concavity of the conditional entropy [1] in $\mathcal{H}_{2} \otimes \mathcal{K}$. The details are as follows: Let $\left(e_{j}\right)$ be an orthonormal basis of $\mathcal{K}$ and consider the operator $\Gamma=\sum_{j}\left(\operatorname{Tr}_{1} A_{j} \rho_{12} A_{j}^{\dagger}\right) \otimes\left|e_{j}\right\rangle\left\langle e_{j}\right|$ on $\mathcal{H}_{2} \otimes \mathcal{K}$. As in the proof of the 2-space theorem we have $\Gamma_{2}=\operatorname{Tr}_{\mathcal{K}} \Gamma=\rho_{2}$ and therefore

$$
H\left(1^{A} \mid 2\right)=S(\Gamma)-S\left(\Gamma_{2}\right) .
$$

This is the conditional entropy of $\Gamma$ with respect to $\mathcal{H}_{2}$. Since $\rho_{12} \mapsto \Gamma$ is linear, the asserted concavity follows from the fact that conditional entropy is concave, as shown in $[1$, Thm. 1]. 
Proof of Theorem 2 (3 space theorem). Lemma 4 implies that $H\left(1^{A} \mid 2\right)+H\left(1^{B} \mid 3\right)$ is a concave function of $\rho_{123}$. Thus, for the proof we may assume that $\rho_{123}$ is a pure state (rank one). In that case $S\left(\rho_{3}\right)=S\left(\rho_{12}\right)$ and, since $B_{k} \rho_{123} B_{k}^{\dagger}$ is pure as well,

$$
H\left(1^{B} \mid 3\right)=-\sum_{k} \operatorname{Tr}_{12} B_{k} \rho_{12} B_{k}^{\dagger} \ln B_{k} \rho B_{k}^{\dagger}-S\left(\rho_{12}\right) .
$$

This reduces the inequality of the 3-space theorem to that of the $2 \frac{1}{2}$-space theorem.

Proof of Theorem 3 ( $2 \frac{1}{2}$ space theorem). The proof runs very parallel to that of the 2space theorem. Namely, the right side in the desired inequality is bounded from below by $\operatorname{Tr}_{12} \rho_{12} \tilde{h}-S\left(\rho_{12}\right)$, where now

$$
\tilde{h}=\mathbb{1} \otimes \ln \rho_{2}-\ln \sum_{j} A_{j}^{\dagger} A_{j} \otimes\left(\operatorname{Tr}_{1} A_{j} \rho_{12} A_{j}^{\dagger}\right)-\ln \sum_{k} B_{k}^{\dagger} B_{k} \rho_{12} B_{k}^{\dagger} B_{k} .
$$

Here we used (6). After applying (5) as before, everything is reduced to showing $\operatorname{Tr}_{12} e^{-\tilde{h}} \leq c_{\infty}^{2}$. The crucial ingredient is again (4) which now leads to

$$
\operatorname{Tr}_{12} e^{-\tilde{h}} \leq \int_{0}^{\infty} d t \sum_{j, k} \operatorname{Tr}_{12} \tilde{C}_{j, k}(t)
$$

with

$$
\tilde{C}_{j, k}(t)=A_{j}^{\dagger} A_{j}\left(\operatorname{Tr}_{1} A_{j} \rho_{12} A_{j}^{\dagger}\right)\left(\rho_{2}+t\right)^{-1} B_{k}^{\dagger} B_{k} \rho_{12} B_{k}^{\dagger} B_{k}\left(\rho_{2}+t\right)^{-1} .
$$

At this point the proof diverges somewhat from that of the 2-space theorem. Namely, by cyclicity of the trace we write

$$
\operatorname{Tr}_{12} \tilde{C}_{j, k}(t)=\operatorname{Tr}_{12}\left(B_{k} A_{j}^{\dagger} A_{j} B_{k}^{\dagger} \tilde{D}_{j, k}(t)\right)
$$

with $\tilde{D}_{j, k}(t)$ given by

$$
\left(\operatorname{Tr}_{1} A_{j} \rho_{12} A_{j}^{\dagger}\right)\left(\rho_{2}+t\right)^{-1} B_{k} \rho_{12} B_{k}^{\dagger}\left(\rho_{2}+t\right)^{-1} .
$$

Since

$$
B_{k} A_{j}^{\dagger} A_{j} B_{k}^{\dagger}\left(\operatorname{Tr}_{1} A_{j} \rho_{12} A_{j}^{\dagger}\right) \leq c_{\infty}^{2}\left(\operatorname{Tr}_{1} A_{j} \rho_{12} A_{j}^{\dagger}\right)
$$

and since $\left(\rho_{2}+t\right)^{-1} B_{k} \rho_{12} B_{k}^{\dagger}\left(\rho_{2}+t\right)^{-1} \geq 0$, we have

$$
\operatorname{Tr}_{12} \tilde{C}_{j, k}(t) \leq c_{\infty}^{2} \operatorname{Tr}_{12} \tilde{D}_{j, k}(t) .
$$

From here, everything is as before:

$$
\sum_{j, k} \operatorname{Tr}_{12} \tilde{D}_{j, k}(t)=\operatorname{Tr}_{2} \rho_{2}^{2}\left(\rho_{2}+t\right)^{-2}
$$

and

$$
\int_{0}^{\infty} d t \sum_{j, k} \operatorname{Tr}_{12} \tilde{D}_{j, k}(t)=\operatorname{Tr}_{2} \rho_{2}=1
$$

This completes the proof. 


\section{Continuous Measurements on Two and Three Spaces}

At last we turn to the continuous version, the most important application being the position-momentum uncertainty. We start with this case. Take $\mathcal{H}_{1}$ to be $L^{2}\left(\mathbb{R}^{d}\right)$, the square-integrable functions on $\mathbb{R}^{d}$. The spaces $\mathcal{H}_{2}$ and $\mathcal{H}_{3}$ can be anything. The measurement $A_{j}^{\dagger} A_{j}$ is $\left|a_{j}\right\rangle\left\langle a_{j}\right|$, where $a_{j}$ is the delta-function $\delta\left(\mathbf{x}-\mathbf{x}^{\prime}\right)$ for some $\mathbf{x}^{\prime}$ in $\mathbb{R}^{d}$. The $j$ becomes $\mathbf{x}^{\prime}$, which is a continuous variable, the sum $\sum_{j}$ becomes the integral $\int d^{d} \mathbf{x}^{\prime}$, and the normalization condition $\sum_{j} A_{j}^{\dagger} A_{j}=1$ becomes $\int d^{d} \mathbf{x}^{\prime} \delta\left(\mathbf{x}-\mathbf{x}^{\prime}\right) \delta\left(\mathbf{x}^{\prime \prime}-\mathbf{x}^{\prime}\right)=$ $\delta\left(\mathbf{x}-\mathbf{x}^{\prime \prime}\right)$. (We realize that the delta-function is not a function, but all of this can be made rigorous.) Similarly, the $B_{k}^{\dagger} B_{k}$ 's are $\left|b_{k}\right\rangle\left\langle b_{k}\right|$, where the $b_{k}$ will also not be squareintegrable functions. They will be plane waves $e^{i 2 \pi \mathbf{k} \cdot \mathbf{x}}$ for some $\mathbf{k}$ in $\mathbb{R}^{d}$. Again, $\mathbf{k}$ is a continuous index and sums are integrals. The normalization condition $\sum_{k} B_{k}^{\dagger} B_{k}=1$ becomes $\int d^{d} \mathbf{k} e^{-2 \pi i \mathbf{k} \cdot \mathbf{x}} e^{2 \pi i \mathbf{k} \cdot \mathbf{x}^{\prime}}=\delta\left(\mathbf{x}-\mathbf{x}^{\prime}\right)$.

Let $\rho_{12}$ be a density matrix on the Hilbert space $L^{2}\left(\mathbb{R}^{d}\right) \otimes \mathcal{H}_{2}$. For every fixed $\mathbf{x} \in \mathbb{R}^{d}$ we can define $\left\langle\mathbf{x}\left|\rho_{12}\right| \mathbf{x}\right\rangle$ as an operator on $\mathcal{H}_{2}$. This really means the partial trace $\operatorname{Tr}_{1}$ of $A_{j}^{\dagger} \rho_{12} A_{j}$. Likewise we define $\left\langle\mathbf{k}\left|\rho_{12}\right| \mathbf{k}\right\rangle$ to be $\operatorname{Tr}_{1} B_{k}^{\dagger} \rho_{12} B_{k}$. We see that $\left\langle\mathbf{x}\left|\rho_{12}\right| \mathbf{x}\right\rangle$ is an operator-valued density in position space and $\left\langle\mathbf{k}\left|\rho_{12}\right| \mathbf{k}\right\rangle$ is the corresponding density in momentum space.

Now we apply the 2-space theorem and infer that

$$
H\left(1^{A} \mid 2\right)+H\left(1^{B} \mid 2\right) \geq S(1 \mid 2),
$$

where

$$
H\left(1^{A} \mid 2\right)=-\int_{\mathbb{R}^{d}} d^{d} \mathbf{x} \operatorname{Tr}_{2}\left\langle\mathbf{x}\left|\rho_{12}\right| \mathbf{x}\right\rangle \ln \left\langle\mathbf{x}\left|\rho_{12}\right| \mathbf{x}\right\rangle-S\left(\rho_{2}\right)
$$

and

$$
H\left(1^{B} \mid 2\right)=-\int_{\mathbb{R}^{d}} d^{d} \mathbf{k} \operatorname{Tr}_{2}\left\langle\mathbf{k}\left|\rho_{12}\right| \mathbf{k}\right\rangle \ln \left\langle\mathbf{k}\left|\rho_{12}\right| \mathbf{k}\right\rangle-S\left(\rho_{2}\right) .
$$

Here $c_{1}=c_{\infty}=\sup _{\mathbf{x}, \mathbf{k}}\left|e^{i 2 \pi \mathbf{k} \cdot \mathbf{x}}\right|=1$ and $\ln c_{1}=\ln c_{\infty}=0$. This is a generalization of the uncertainty principle in [8], which is what (8) reduces to when $\rho_{12}=\rho_{1} \otimes \rho_{2}$ is a product state. The 3 -space theorem and the $2 \frac{1}{2}$-space theorem obviously generalize in a similar way for the Fourier transform.

This example of a continuum version of our theorems has the obvious generalization to positive operator-valued measures (POVMs). The interested reader can work this out for him/herself, but we mention here one further specific generalization. Let us take $\mathcal{H}_{1}=L^{2}(X, d \mathbf{x})$, where $X$ is some configuration space (e.g., $X=\mathbb{R}^{d}$ or $X=$ a torus or $X=$ a lattice) and $L^{2}(X, d \mathbf{x})$ are the square integrable functions on $X$ with respect to some measure $d \mathbf{x}$. The measurements $A_{j}^{\dagger} A_{j}$ are again given by rank one projections corresponding to the functions $\delta_{\mathbf{x}^{\prime}}(\mathbf{x})$ for some $\mathbf{x}^{\prime}$ in $X$. Now let $\mathcal{H}_{1}^{\prime}$ be a second Hilbert space of the form $L^{2}(K, d \mathbf{k})$ and let $\mathcal{U}$ be a unitary from $\mathcal{H}_{1}$ to $\mathcal{H}_{1}^{\prime}$, which is given by a $\operatorname{kernel} \mathcal{U}(\mathbf{k}, \mathbf{x})$. We assume that $\operatorname{ess}^{\sup _{\mathbf{k}, \mathbf{x}}}|\mathcal{U}(\mathbf{k}, \mathbf{x})|$ is finite, which is the same thing as assuming that $\mathcal{U}$ is a bounded operator from $L^{1}(X, d \mathbf{x})$ to $L^{\infty}(K, d \mathbf{k})$ with

$$
\|\mathcal{U}\|_{L^{1}(X, d \mathbf{x}) \rightarrow L^{\infty}(K, d \mathbf{k})}=\sup _{\|f\|_{L^{1}(X, d \mathbf{x})}=1,\|g\|_{L^{\infty}(K, d \mathbf{k})}=1}|\langle g, \mathcal{U} f\rangle|=\operatorname{ess}_{\sup }|\mathcal{k}, \mathbf{U}(\mathbf{k}, \mathbf{x})| .
$$


For each $\mathbf{k} \in K$, we can think of $\mathcal{U}(\mathbf{k}, \mathbf{x})$ as a function of $\mathbf{x}$ and we define the $B_{k}^{\dagger} B_{k}$ 's to be the rank-one projections onto these functions. In this way we obtain, as before, operators $\left\langle\mathbf{x}\left|\rho_{12}\right| \mathbf{x}\right\rangle$ and $\left\langle\mathbf{k}\left|\rho_{12}\right| \mathbf{k}\right\rangle$ on $\mathcal{H}_{2}$. Now $H\left(1^{A} \mid 2\right)$ and $H\left(1^{B} \mid 2\right)$ are defined as in the Fourier transform case, except that the integration is over the sets $X$ and $K$, respectively.

Theorem 5 (Generalized Fourier transform). Inequality (3) is valid with

$$
c_{1}=\operatorname{ess} \sup _{\mathbf{k}, \mathbf{x}}|\mathcal{U}(\mathbf{k}, \mathbf{x})| .
$$

An example in which the classical entropies are simultaneously discrete and continuous is the following. Suppose $X=\mathbb{Z}$, i.e., the integers, like the sites in a tight binding model. An $L^{2}$ function is the wave function of an itinerant electron. The second space $\mathcal{H}_{1}^{\prime}$ is the square integrable functions on $K=(-1 / 2,1 / 2)$, the Brillouin zone. In this case the delta-functions, $\delta_{x x^{\prime}}$, on $X$ are legitimate Kronecker deltas, and the (normalized) plane waves on $X$, parametrized by $k \in K$, are $e^{2 \pi i k x}$. The unitary here is $\mathcal{U}(k, x)=e^{2 \pi i k x}$ and therefore $c_{1}=1$. Since $d x$ is counting measure on $\mathbb{Z}$ the expression of $H\left(1^{A} \mid 2\right)$ is a sum $\sum_{x \in \mathbb{Z}}$, whereas $d k$ is ordinary Lebesgue measure on $(-1 / 2,1 / 2)$ and $H\left(1^{B} \mid 2\right)$ is an integral $\int_{K} d k$.

Conclusion. We have shown several things. (1) The entropy inequalities in [12,7] can be proved in a few lines essentially by imitating the original proof of strong subadditivity of entropy. (2) We have carried at least one of these inequalities forward by utilizing the trace norm $c_{1}$ instead of the operator norm $c_{\infty}$. (3) We have shown how these inequalities, suitably interpreted, extend the 2-space entropy uncertainty principle to continuous bases such as position and momentum.

Acknowledgement. We thank Zhihao Ma for helpful correspondence. U.S. National Science Foundation grants PHY-1068285 (R.F.) and PHY-0965859 (E.L.) and the Simons Foundation grant 230207 (E.L.) are acknowledged.

\section{References}

1. Lieb, E.H., Ruskai, M.B.: Proof of the strong subadditivity of quantum-mechanical entropy. J. Math. Phys. 14(12), 1938-1941 (1973)

2. Maassen, H., Uffink, J.B.M.: Generalized entropic uncertainty relations. Phys. Rev. Lett. 60, 1103 (1988)

3. Hirschman, I.I. Jr.: A note on entropy. Amer. J. Math. 79, 152-156 (1957)

4. Deutsch, D.: Uncertainty in quantum measurements. Phys. Rev. Lett. 50, 631-633 (1983)

5. Kraus, K.: Complementary observables and uncertainty relations. Phys. Rev. D 35, 3070-3075 (1987)

6. Wehner, S., Winter, A.: Entropic uncertainty relations - a survey. New J. Phys. 12, 025009 (2010)

7. Coles, P.J., Yu, L., Gheorghiu, V., Griffiths, R.B.: Information-theoretic treatment of tripartite systems and quantum channels. Phys. Rev. A 83, 062338 (2011)

8. Frank, R.L., Lieb, E.H.: Entropy and the uncertainty principle. Ann. H. Poincaré 13(8), 1711-1717 (2012)

9. Rumin, M.: An entropic uncertainty principle for positive operator valued measures. Lett. Math. Phys. 100(3), 291-308 (2012)

10. Hall, M.J.W., Wiseman, H.M.: Heisenberg-style bounds for arbitrary estimates of shift parameters including prior information. New J. Phys. 14, 033040 (2012)

11. Renes, J.M., Boileau, J.-C.: Conjectured strong complementary information tradeoff. Phys. Rev. Lett. 103, 020402 (2009)

12. Berta, M., Christandl, M., Colbeck, R., Renes, J.M., Renner, R.: The uncertainty principle in the presence of quantum memory. Nature (Physics) 6, 659-662 (2010)

13. Tomamichel, M., Renner, R.: Uncertainty relations for smooth entropies. Phys. Rev. Lett. 106, 110506 (2011)

14. Coles, P.J., Colbeck, R., Yu, L., Zwolak, M.: Uncertainty relations from simple entropic properties. Phys. Rev. Lett. 108, 210405 (2012) 
15. Lieb, E.H.: Convex trace functions and the Wigner-Yanase-Dyson conjecture. Adv. in Math. 11, 267-288 (1973)

16. Lindblad, G.: Expectations and entropy inequalities for finite quantum systems. Commun. Math. Phys. 39, 111-119 (1974)

17. Carlen, E.A.: Trace inequalities and quantum entropy. An introductory course. In: Entropy and the quantum, R. Sims, D. Ueltschi (eds.), Contemp. Math. 529, Providence, RI: Amer. Math. Soc., 2010, pp. 73-140

18. Davis, C.: A Schwarz inequality for convex operator functions. Proc. Amer. Math. Soc. 8, $42-44$ (1957)

Communicated by M. Aizenman 